\title{
A Dynamic Resource Scheduling Algorithm For OFDM System
}

\author{
Liang Xiao, Anchun Wang, Shidong Zhou, Yan Yao \\ State Key Lab on Microwave and Digital Communications \\ Tsinghua University, Beijing 100084, China \\ E-mail: xiaoliang00@mails.tsinghua.edu.cn
}

\begin{abstract}
This article presents a practical scheduler algorithm Multiple Carrier Proportional Fairness (MCPF), to dynamically allocate resource for the Orthogonal Frequency Division Multiplex (OFDM) system in both frequency and time domain. We analyze its performance on the Doppler fading multi-path channel with CoCentric System Studio and find that the throughput of MCPF is more than $60 \%$ greater compared with the Fixed Scheduling algorithm when there are more than 2 users. This algorithm satisfies proportional fair rule and its throughput performance increases with the number of system user, reaching $100 \%$ in 8 users system.
\end{abstract}

Keywords - Dynamic Resource Scheduling, MCPF, OFDM, CCSS

\section{INTRODUCTION}

The demand on high rate multimedia information has pushed the development of wireless communication systems in an unprecedented pace. System design of fourth-generation (4G) technology has already begun, which seeks to support a wide range of packet services with highest data rates reaching 20Mbps. With limited bandwidth resources, efficient scheduling algorithm has to be put up to provide satisfactory services to the users.

Multimedia information sources not only exhibit highly bursty traffic rates, but also have variable quality of service (Qos) requirement and traffic rate characteristics. It is especially challenging to develop multimedia packet scheduling algorithm in the wireless systems because of its bursty errors, scarce bandwidth, location-dependent and timevarying link capacity and etc. Some wireless scheduling algorithms have already been proposed, such as idealized wireless fair queueing (IWFQ), channel-condition independent packet fair queueing (CIF-Q), server-based fair approach (SBFA), all based on the assumption of two-state Markov channel [1]. In these algorithms, the wireless link has full capacity in error-free state and has zero capacity in error state. However, it is not the case in the real wireless transmission, which has multiple states link and different physical capacity.

Considering this, the CDMA/HDR (High Data Rates) introduced a dynamic data rate transmission technique allowing efficient data transmission with variable data rate according to the channel condition of each MT. Accordingly, its scheduling adopts the DRC/R algorithm shown in [2], a practical way to achieve proportional fairness (PF) that maximizes channel throughput with fairness constraint. Based on their work, A. Jalali et al. made an analysis to estimate its forward link throughput [3] and Jong H.R et al. proposed a WFQ-PF algorithm to balance throughput and delay differences between users with different location [4].

OFDM is also an important candidate technique for the future wireless transmission. But its multi-carrier nature makes its scheduler different from that of CDMA/HDR, which allocates resource in only time domain. The existing researches for OFDM maximize the system capacity, lower transmission power, or lower BER (Bit Error Rate) by optimizing the allocation of the subcarrier, bit and power in OFDM [5][6][7][8]. But both their algorithm complexity and system spending are too large. Therefore in this paper we present a dynamic scheduling scheme for OFDM system Multiple Carrier Proportional Fairness (MCPF), satisfying the proportional fairness (PF).

In each slot of MCPF, we divided $\mathrm{M}$ sub-carrier into $\mathrm{N}$ subbands, which are made up of $\mathrm{M} / \mathrm{N}$ neighbouring subcarriers and is the minimum resource unit to be allocated. In each subband, the $\mathrm{DRC} / \mathrm{R}$ algorithm presented in [2] is adopted, where the Data Rate Control (DRC) is determined by the measured Signal Noise Rate (SNR) of that subband and is transmitted from the access terminal (AT) to the access point (AP). AP will allocate this subband to the user with the highest DRC/R. After surfing all N subbands, MCPF unified the coding and modulation process of the same user in different subbands in order to achieve a larger interleave gain. The Adaptive Modulation Coding (AMC) is utilized under the control of MCPF in our simulation system, which is built on the Synopsys's CoCentric System Studio (CCSS). On the channel considering both six-path Multipath fading and Doppler fading, we find that MCPF can improve system throughput in the multi-user systems compared with a static scheduling algorithm called Fixed Scheduling (FS).

In Section II, we illustrate two OFDM resource allocation algorithms MCPF and FS separately. In Section III, simulation model is built and simulation results are presented and discussed. In Section IV, we present our future research 
plan. Finally, a brief summary of this paper is made in Section V.

\section{SCHEDULING ALGORITHMS FOR OFDM}

We now discussed the resource scheduler in the downlink multi-user packet transmission for OFDM system, which not only decides the users to be served in each slot, but also the modulation and coding scheme in all subbands. Two scheduling schemes will be presented in the next paragraph: $\mathrm{MCPF}$ and its comparison, FS.

In both schemes, AP buffers the data of each user in a queue and carries out the scheduling for these data in the minimum allocation resource unit called subband per slot. As to the AT, it measures M sub-carrier' SNR from the PILOT signal and N subband's SNR are seen as the average value of $\mathrm{M} / \mathrm{N}$ SNRs in the subband. The DRC is determined by subband SNR with the transmission scheme in table 1, which can achieve BER less than 1\%. Each AT feedback several high DRCs as well as the corresponding subband Ids to AP and these are the basis of the scheduling. Adaptive Modulation Coding is utilized in the simulation system, which can realize encoding process with any code rate by puncture.

\section{A. MCPF Algorithm}

MCPF utilizes the proportional fair rule in the OFDM system, balancing the relationship between system throughput and user fairness. The algorithm works as follows:

1. Determine and feedback DRC (AT).

2. Select user for each subband: The user with the highest ratio of $D R C_{i}^{j} / R_{i}$ that applies for this subband will be selected for this subband, where $D R C_{i}^{j}$ denotes the DRC of the ith-user at the jth-subband and $R_{i}$ is the average rate received by the ith-user. A user may obtain more than one resource units and suppose the ith-user obtains $\mathrm{k}$ subbands in this slot.

3. Determine the transmission schemes for each user: All subbands for the same user are unified in the coding and modulation processes to obtain a larger interleave gain. The modulation parameter is the highest value from $\mathrm{k}$ subbands and the overall transmission data size $\mathrm{L}$ is the sum of $\mathrm{k}$ DRCs.

4. Update Average Rate of each user: For the ith-user,

$$
R_{i}=\left(1-1 / T_{c}\right) R_{i}+1 / T_{c} * L
$$

, where Tc inherits the meaning in DRC/R algorithm in [2], related to the maximum time to be starved. For the user that is not served by any subband, its $\mathrm{R}$ should be updated as

$$
R_{i}=\left(1-1 / T_{c}\right) R_{i}
$$

The influence of Tc on system throughput will be discussed later.

\begin{tabular}{|r|l|l|l|l|}
\hline $\begin{array}{l}\text { Class } \\
\text { ID }\end{array}$ & $\begin{array}{l}\text { C/I } \\
(\mathrm{dB})\end{array}$ & $\begin{array}{l}\text { Code } \\
\text { Rate }\end{array}$ & $\begin{array}{l}\text { Modulate } \\
\text { Type }\end{array}$ & $\begin{array}{l}\text { DRC } \\
\text { (bits/slot) }\end{array}$ \\
\hline 1 & -3.4 & $1 / 4$ & BPSK & 128 \\
\hline 2 & -0.4 & $1 / 2$ & BPSK & 256 \\
\hline 3 & 2.2 & $1 / 2$ & QPSK & 512 \\
\hline 4 & 5.2 & $3 / 4$ & QPSK & 768 \\
\hline 5 & 7.6 & $2 / 3$ & $8 \mathrm{PSK}$ & 1024 \\
\hline 6 & 10.9 & $3 / 4$ & $16 \mathrm{QAM}$ & 1536 \\
\hline 7 & 14.5 & $2 / 3$ & $64 \mathrm{QAM}$ & 2048 \\
\hline
\end{tabular}

Table 1. Transmission Scheme in simulation

\section{B FS Algorithm}

To evaluate MCPF, we use FS algorithm shown in figure 1. In this algorithm, the subband is given to a fixed user. When the number of subband is greater than that of user, the jthsubband is allocated to the user whose ID equals to $\mathrm{j}$ mod 16 at any slot. Its modulation and coding parameters are also related to the user's downlink SNR at this subband. Otherwise, each user will be allocated to the fixed subband in a fixed period more than one slot.

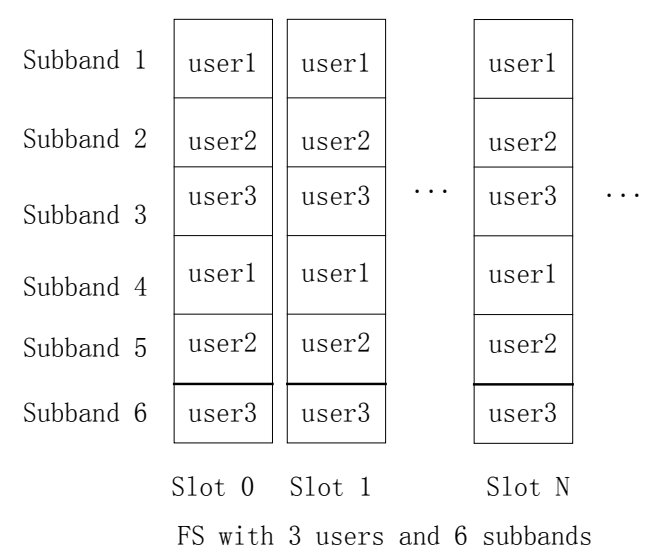

Figure 1. FS algorithm

\section{SIMULATION MODEL AND RESULTS}

The performance of MCPF and FS is analyzed and compared through the computer simulation with Synopsys's CCSS. In the simulation, there are 1024 sub-carriers and 16 subbands for the OFDM system. The carrier frequency is $5 \mathrm{GHz}$ and the symbol duration is $100 \mathrm{~ns}$. There are 8 symbols 
in each sub-carrier per slot. We assume double over-sampling rate and set the sample rate of receive signal to be $20 \mathrm{M} / \mathrm{s}$.

In the forward link multi-user simulation system, there are one AP and one to eight users, each in an AT moving with the velocity of $20 \mathrm{mps}$ from the same locations to the AP. Thus the channel can be simplified into a model considering only Multi-path fading and Doppler fading. We adopt the six paths model shown in table 1 and the average receive SNR is set to $5 \mathrm{~dB}$.

\begin{tabular}{|l|l|l|}
\hline Tap\# & Delay [us] & Power $[\mathrm{dB}]$ \\
\hline 1 & 0 & 0 \\
\hline 2 & 0.31 & -1 \\
\hline 3 & 0.71 & -9 \\
\hline 4 & 1.09 & -10 \\
\hline 5 & 1.73 & -15 \\
\hline 6 & 2.51 & -20 \\
\hline
\end{tabular}

Table 2. Channel Parameters

Figure 2 compares the throughputs of FS and MCPF when the number of user ranges from 1 to 8 and Tc equals to 200. This figure indicates that the average throughput of $\mathrm{MCPF}$ is greater than that of FS by more than 60 percent when $\mathrm{NU}$, the number of user is greater than two. Further on, MCPF's performance on system throughput increases evidently with the number of user before it reaches 7 but this increase becomes slight afterwards. With more users, there is more possibility for MCPF to find a user with better channel condition at the subband. Therefore, MCPF has throughput advantage in the multi-user systems.

Figure 3 describes the average throughput of MCPF for each user when $\mathrm{Tc}=200$ and $\mathrm{NU}=4$. It is shown that there is no evident priority for any of the four users. Thus the system resource can be allocated relatively fairly to the users using MCPF.

Figure 4 illustrates the slow increase of system throughput with Tc. When Tc rises from 1 to 2000, only $10.3 \%$ throughput gain is achieved. This can be explained with equation (1). First, we can see $\mathrm{R}$ is constant if $T_{c}=\infty$. So, the allocation is decided solely by instantaneous $\mathrm{DRC}$, leading to maximum system throughput and poor fairness characteristics. On the other hand, when $\mathrm{Tc}=1, \mathrm{DRC} / \mathrm{R}$ is approximately a constant and thus the scheduling becomes fair and random. Therefore, Tc means the trade-off between fairness and throughput and the turning point in the figure, $\mathrm{Tc}=200$ is a good choice.

\section{FUTURE RESEARCH}

There is still large research space for MCPF. Firstly, we will further study the influence of user location on the algorithm. A large number of users with different topology and different velocity will be simulated and we believe some interesting results will be achieved. Secondly, the impact of the minimum allocation resource partition on the system throughput is still under research. And how to encode the feedback DRCs and their subband ID is another interesting topic waiting for discussion.

\section{CONCLUSIONS}

We presented a practical dynamic resource allocation scheme-MCPF for the OFDM system that satisfies the proportional fairness. Through simulation on the Multipath and Doppler fading channel with CCSS, we found that despite its low algorithm complexity, MCPF can achieve better performance in both fairness and system throughput compared with FS, a static scheduling algorithm. The advantage of MCPF in throughput increases with the number of user. Afterwards, we discussed the influence of parameter Tc on the algorithm performance. In the end of the paper, we gave a brief expectation of our future research.

\section{REFERENCES}

[1] Yaxin Cao and Victor O. K. LI scheduling algorithms in broad-band wireless networks, IEEE Proceedings of the IEEE, 2001.1.

[2] P.Bender et al. CDMA/HDR:A Bandwidth-Efficient High-Speed Wireless Data Service for Nomadic Users, IEEE Communications Magazine, July 2000

[3] A.Jalali, R.Padovani, R.Pankaj, Data Throughput of CDMA-HDR a High Efficiency-High Data Rate, Personal Communication Wireless System, VTC 2000-Spring Tokyo, pp.1854-1858 vol.3.

[4] Jong Hun Rhee, A Wireless Fair Scheduling Algorithm for 1 x EV-DO System, VTC 2001, pp.743-746.

[5] L.Piazzo: Fast algorithm for power and bit allocation in OFDM systems. ELECTRONICS LETTERS ,9th December 1999, Vol 35, No 25,pp2173 2174.

[6] Lai.S.K,Cheng R.S and Letaief K.B: Adatpive trellis coded MQAM and power optimization for OFDM transmission. Proc. IEEE VTC Spring ,Houston, 1999.

[7] Lai S.K, Cheng R.S, Letaief K.B and Chi Ying Tsui: Adaptive Tracking of Optimal Bit and Power Allocation for OFDM Systems in Time-Varying Channels. ISBN: 0-7803-5668-3,1999,pp776 780.

[8] D. A. Gore, R. U. Nabar and A. Paulraj, "selecting an optimal set of transmit antennas for a low rank matrix channel”, in Proc. ICASSP'00, Istanbul, Turkey, pp.2785-2788, June 2000. 


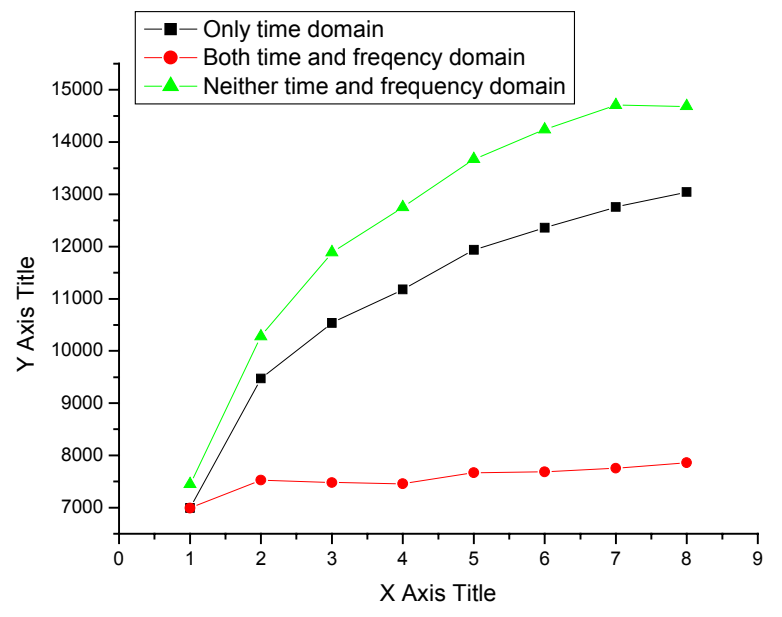

Figure 2. MCPF vs. FS in average throughput when $\mathrm{Tc}=200$.

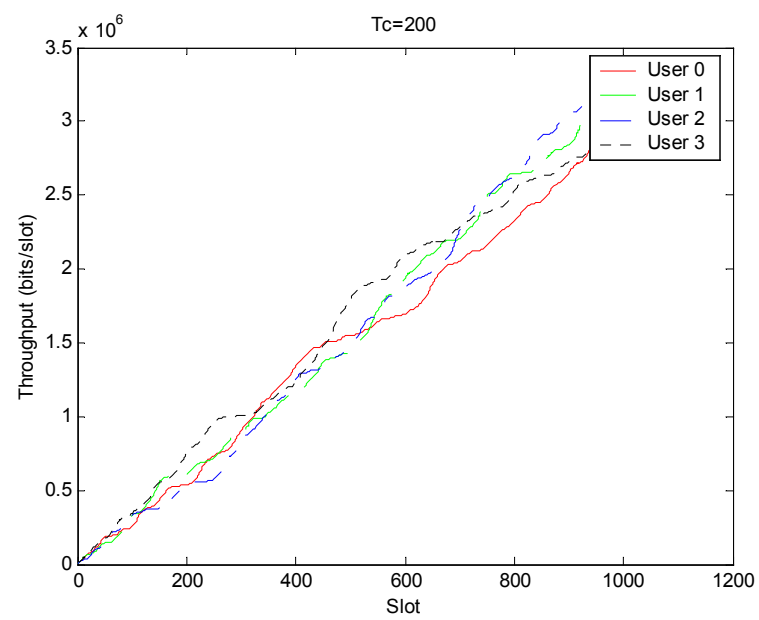

Figure 3. Average throughputs of four users when $\mathrm{Tc}=200$.

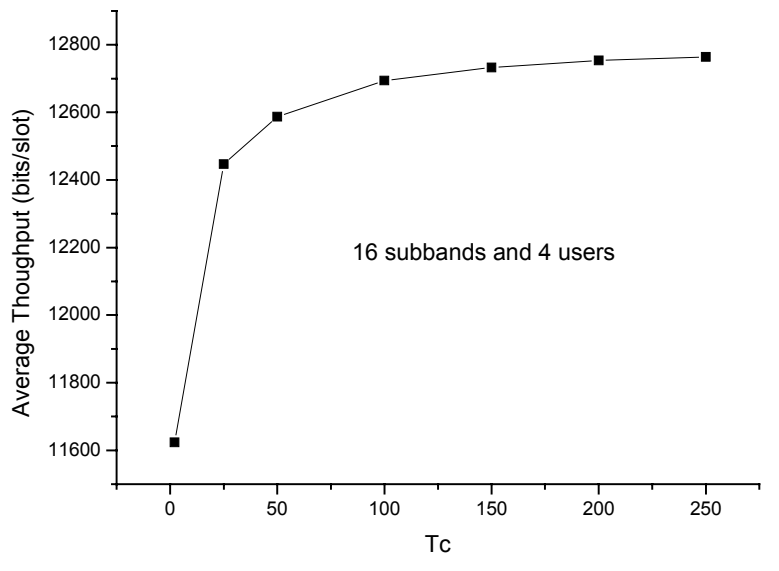

Figure 4. Average throughput when the number of user is 4. 